\title{
Language-independent working memory: Evidence from German and French reading span tests
}

\author{
MARIKO OSAKA \\ Osaka University of Foreign Studies, Osaka, Japan \\ NAOYUKI OSAKA \\ Kyoto University, Kyoto, Japan \\ and \\ RUDOLF GRONER \\ University of Bern, Bern, Switzerland
}

\begin{abstract}
The efficiency of working memory was measured with two versions of reading span tests, written in German and in French, using German/French bilingual subjects. The correlation between German and French versions was found to be highly significant $(r=0.85)$. Our results indicate that the efficiency of working memory for reading is independent of language, reconfirming the results obtained by Osaka and Osaka (1992).
\end{abstract}

The reading span test (RST) is a test designed to measure the efficiency of working memory during reading (Daneman \& Carpenter, 1980). The RST can measure the working-memory capacity that is closely related to reading ability of individuals (Just \& Carpenter, 1992). Good readers are assumed to be efficient in reading the text; they need not waste the capacity allocated for processing the information, and they can store the products of the processes easily.

Osaka and Osaka (1992) showed that the working memory measured with Japanese and English RSTs was language independent. Their subjects were Japanese students who had studied English for 7-9 years. The correlation between the two languages was significantly high; a reader who got a high span in the first language also got a high span in the second language.

To further reconfirm the language independence of working memory, we compared the RST scores of German and French versions of the RST using German/ French bilingual subjects. In addition, we examined the age effect under the hypothesis that working-memory efficiency is inversely correlated with increasing age.

\section{METHOD}

\section{Subjects}

The subjects were 15 Swiss students and staff members from the University of Bern who were 23 to 50 years old. They were all bilingual

This work was supported in part by a grant from the Japan Society for the Promotion of Science under the International Project on Comparative Reading Behavior and by Grant 03801010 from the Ministry of Education to the second author. Correspondence should be addressed to N. Osaka, Department of Psychology, Faculty of Letters, Kyoto University, Kyoto 606 , Japan. in German and French. They were all native speakers of Swiss German, but they had 7-9 years' learning experience of French and used both languages frequently in their daily lives.

\section{Materials}

Seventy German sentences and 70 French sentences were selected for the German and French RSTs, respectively. The sentences were 10-16 words long. Before the experiment, both the German and French sentences were evaluated by the German/French bilingual subjects. The difficulty levels of both groups of sentences were found to be almost the same. The difficulty levels of the sentences were controlled.

\section{Procedure}

The procedures of these RSTs were the same as the English version (Osaka \& Osaka, 1992). Each sentence was printed on a single line across the center of a $13 \times 18 \mathrm{~cm}$ white card. The cards were arranged in five sets, each of which comprised two, three, four, and five sentences. Blank cards were inserted between the sets. Within a set, the sentences were not related to each other. The subject was asked to read each sentence aloud at his/her own pace. As soon as the subject finished reading one sentence, the next sentence was presented and the subject was to immediately read it aloud too, and so on. After reading all the sentences in a set, the subject was asked to recall the last word of each sentence within the set. The order of reporting these final words was based on the free-recall procedure. To avoid the recency effect, the subject was not allowed to report the last target word first within each set. The subject was presented with increasingly longer sets of sentences until he/she failed four sets at a particular level. The level at which a subject correctly answered three out of five sets was taken as a measure of the subject's reading span. The subject was asked to recall the last word of each sentence within the set in both German and French RSTs.

\section{RESULTS AND DISCUSSION}

The reading span of the 15 subjects on the German RST varied from 3.0 to $5.0(M=3.80, S D=0.56)$ and on the French RST from 2.0 to $4.0(M=2.93, S D=0.50)$. The German reading spans are higher than the original English reading spans $(M=3.15)$ of Daneman and Carpenter (1980) and the results obtained by Osaka and Osaka 


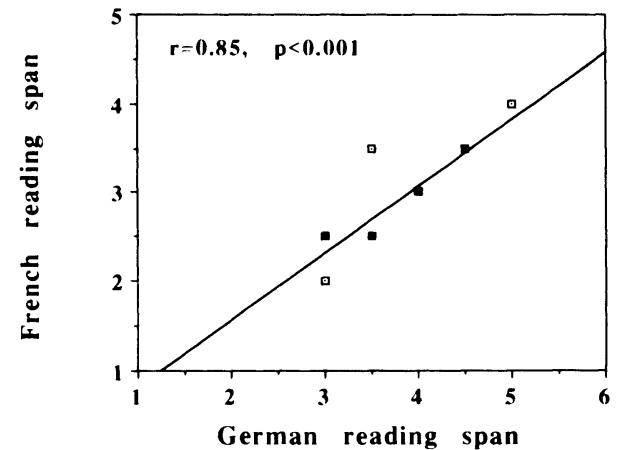

Figure 1. Correlation between the German and French versions of RST scores. Filled squares show the same scores measured more than two times $(n=15)$.

(1992) in the Japanese RST $(M=3.45)$. One possible reason for the high span scores in the German RST is most likely that some of the last words in the German sentences were the basic parts of the verbs and auxiliary verbs; for example, ein or haben. This made it easier for the subjects to recall the target words in the German RST. The French reading spans are very close to the results of the CMU version of Osaka and Osaka $(M=2.88)$. Most of the subjects got higher scores in the German version than in the French version. There were significant differences between the German and French versions of the RST $(t=$ $8.975, p<.001)$. Only one subject who got equal scores (3.5 for both German and French RSTs) is completely bilingual; her father is a native German speaker, and her mother is a native French speaker. Therefore, she grew up using two languages equally.

Figure 1 shows a high correlation between the German and French versions of the RST. The correlation coefficient between the German and French RSTs was 0.85 $(p<.001)$. The correlation coefficient of this study is nearly equivalent to previous results using Japanese and English (ESL version) RSTs $(r=0.84)$. This result confirmed the results obtained by Osaka and Osaka (1992). The correlation between the first language (L1) and the second language (L2) was found to be significantly high, and the working-memory capacity related to two-language processing was independent of language. If a reader has a high reading span in $\mathrm{L} 1$, then he can also get a high reading span in $L 2$.

We also found an age difference in RSTs. The RST scores of older subjects were lower than those of younger subjects. The correlation coefficient between age and the German RST score was $-0.54(p<.05)$. The correlation between age and the French RST score, however, was not significant $(-0.45, p<.09)$. This indicates that as age increased, the RST score decreased. The result is in good agreement with a previous investigation by Salthouse and Babcock (1991), in which significant negative correlations between age and measures of working memory were found.

These results suggest that the span measured with the RST is closely related to how effectively a subject uses working memory while he/she reads the sentences.

\section{REFERENCES}

Daneman, M., \& Carpenter, P. A. (1980). Individual differences in working memory and reading. Journal of Verbal Learning \& Verbal Behavior, 19, 450-466.

Just, M. A., \& CARPENTER, P. A. (1992). A capacity theory of comprehension: Individual differences in working memory. Psychological Review, 99, 122-149.

OSAKA, M., \& OSAKA, N. (1992). Language-independent working memory as measured by Japanese and English reading span tests. Bulletin of the Psychonomic Society, 30, 287-289.

Salthouse, T. A., \& BABCock, R. L. (1991). Decomposing adult age differences in working memory. Developmental Psychology, 27, 763-776.

(Manuscript received September 25, 1992.) 